\title{
Multivariate Statistical Approaches for Electron Backscattered Diffraction
}

\author{
L.N. Brewer, P.G. Kotula, J.R. Michael
}

Sandia National Laboratories, Materials Characterization Dept., P.O. Box 5800-MS 1411 Albuquerque, NM 87185-1411

Electron backscattered diffraction (EBSD) is a widely used technique for both identifying the crystallographic phase and for mapping the orientation of crystalline materials on the micron length scale.[1] Often the operating conditions necessary for phase identification are not suitable for orientation mapping and vice versa. In an effort to optimize the speed involved in the mapping technique, pattern quality is sacrificed and the wealth of information present in an EBSD pattern is compressed to basically 4 values: a matched phase and three Euler angles. However, ab initio identification of phases from EBSD patterns requires high quality patterns and fairly intense computation. [2]

Spectrum imaging is an analytical approach that may offer some solutions to the aforementioned problems. Spectrum imaging consists of collecting a whole spectrum at each pixel in a mapping style measurement. This large set of data is then analyzed using multivariate statistical analysis (MSA) techniques such as principle components analysis, multivariate curve resolution, or other least squares based techniques. The result of these calculations is a set of component spectral shapes with corresponding abundances that allow the analyst to extract the greatest amount of physically relevant information from an otherwise enormous data set. Spectrum imaging has been used successfully in EDX microanalysis[3] (both in the SEM and TEM), TOF-SIMS, WDS, and EELS.

To examine the potential benefits of the spectrum imaging approach for EBSD data, a series of basic experiments and calculations were run. Test data sets (20x20 patterns in .jpeg format) on polycrystalline $\mathrm{Al}$ and on the directionally solidified eutectic oxide, $\mathrm{CoO} / \mathrm{ZrO}_{2}(\mathrm{CaO})$, were collected using the HKL Channel 5 system with a Nordlys detector under normal mapping conditions. The data was collected on a FEI dual beam FIB (model DB235) and a Zeiss (Supra 55 VP) SEM at $20 \mathrm{keV}$ for $\mathrm{Al}$ and $\mathrm{CoO} / \mathrm{ZrO}_{2}(\mathrm{CaO})$, respectively. The data sets were analyzed according to the schematic shown in Figure 1. Each EBSD pattern was hough transformed, unzipped into a 1-D vector of channels with intensities ranging from $0-255$, and then added to an overall data matrix. A range of treatments (edge/no edge detection, spatial simplicity/spectral simplicity, etc.) were examined to determine the optimal way of treating the data. The multivariate analyses were performed using the AXSIA code developed at Sandia National Laboratories. [3]

The MSA techniques were able to correctly identify individual grains in the Al sample and individual phases in the $\mathrm{CoO} / \mathrm{ZrO}_{2}(\mathrm{CaO})$ sample. For each component EBSD pattern identified from the Al data, a corresponding color map of abundance can be seen which clearly corresponds to a single grain (Figure 2). The success in the $\mathrm{CoO} / \mathrm{ZrO}_{2}(\mathrm{CaO})$ sample is particularly notable due to both phases sharing the Fm-3m space group which would confuse most autoindexing routines. The range of analytical treatments identified two extremes in results: a minimal number of components (patterns) with only kikuchi line positions present or a larger number of components with full intensity information present. The further application of these results to phase mapping will be discussed. 
1. A. J. Schwartz, et al., Electron Backscatter Diffraction in Materials Science. 2000, New York: Kluwer Academic.

2. J. R. Michael and R. P. Goehner. Electron backscatter diffraction: A powerful tool for phase identification in the SEM. in MRS Fall Meeting. 2001. Boston, MA: MRS.

3. P. G. Kotula, et al., Microscopy and Microanalysis. 9(1):(2003) p. 1-17.

4. Sandia is a multiprogram laboratory operated by Sandia Corporation, a Lockheed Martin Company, for the United Stated Department of Energy (DOE) under contract DEAC0494AL85000.

Figure 1. Schematic of multivariate statistical analysis of EBSD data set.

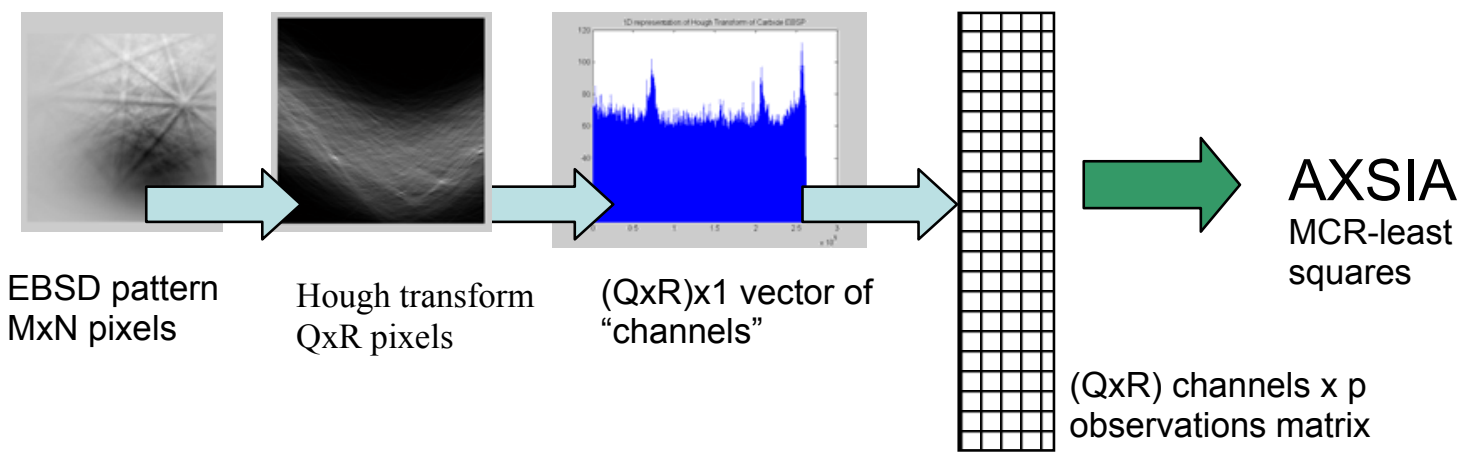

Figure 2. A.) EBSD pattern components from MSA calculations with edge detection prior to hough transform, B.) Corresponding abundance map for each component, C.) Composite map of component abundances.

A.
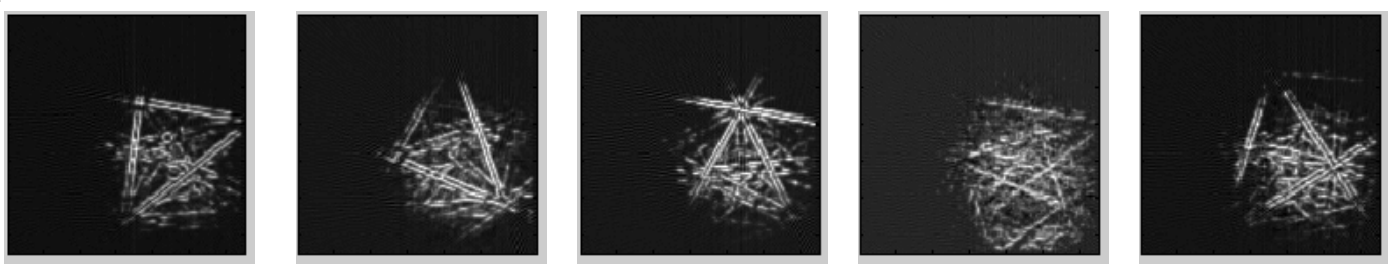

B.
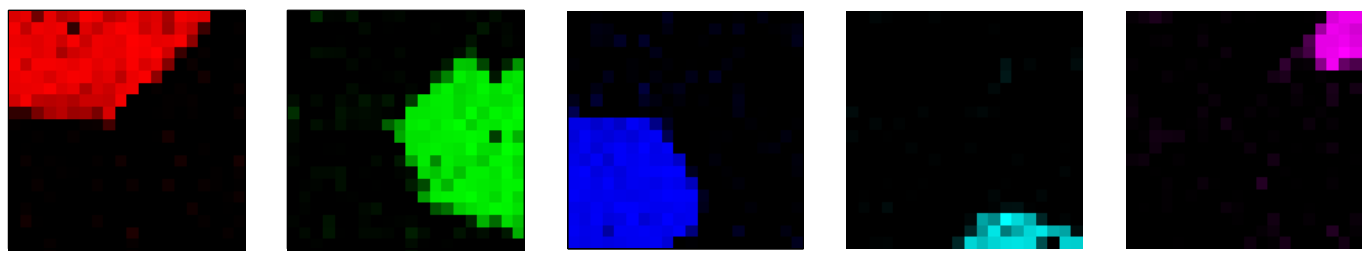

C.

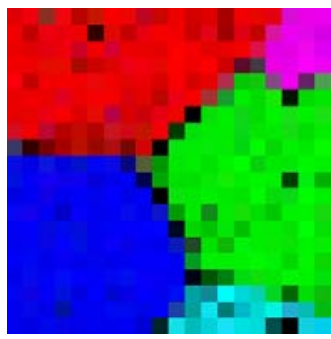

